\title{
Social Business in der Praxis
}

\section{Mit welchen Herausforderungen zu rechnen ist}

\author{
Christian Zinke-Wehlmann (i), Julia Friedrich (1), Mandy Wölke und \\ Vanita Römer
}

\subsection{Das Projekt SB:Digital}

Social Media bilden die Grundlage für grenzenlose Vernetzung und Interaktion basierend auf digitaler Technologie. Während für Privatpersonen der Nutzen u. a. in der Kontaktpflege mit Freunden weltweit sowie Meinungsäußerung zu dem generierten Content besteht (z. B. durch Likes oder Posts), stehen für Unternehmen hier andere Dinge im Vordergrund. Neben den klassischen marketinggetriebenen Kommunikationszielen zur Kundengewinnung und Kundenbindung, nutzen Unternehmen vermehrt auch Expertennetzwerke zur Informationsgewinnung oder werben neue Mitarbeitende über berufliche Netzwerke (Xing) an. Daneben spielt inzwischen aber auch die Vernetzung innerhalb von Unternehmen eine zunehmende bedeutende Rolle. Die Nutzung sozialer Medien innerhalb von Unternehmen oder Unternehmensnetzwerken (in der Literatur auch als Enterprise Social Network (ESN) bezeichnet) bietet erhebliche produktionssteigernde Potenziale, die laut des McKinsey Global Institute bis dato ungenutzt sind. Das Forschungsprojekt SB:Digital schließt diese Lücke. Neben dem Bereich der Kommunikation eröffnen soziale Netzwerke enormes Potenzial für den Bereich Wissensmanagement, Innovationsmanagement und Partizipation [5]. Sie können dabei helfen, effizientere Verbindungen zwischen den Mitarbeitenden zu schaffen und Informationen sowie Daten unkompliziert untereinander auszutauschen [3]. Zur Erfassung der Effekte,

C. Zinke-Wehlmann $(\bowtie)$

Universität Leipzig, Universitätsrechenzentrum, Leipzig, Deutschland

J. Friedrich · M. Wölke · V. Römer

Institut für Angewandte Informatik e.V. an der Universität Leipzig, Leipzig, Deutschland

(C) Der/die Autor(en) 2021

W. Bauer et al. (Hrsg.), Arbeit in der digitalisierten Welt,

https://doi.org/10.1007/978-3-662-62215-5_24 
Gestaltungsparameter und Möglichkeiten des Nutzens von sozialen Netzwerken im unternehmensinternen Gebrauch wurde das Konzept „Social Business“ im Rahmen des Verbundprojektes untersucht und weiterentwickelt. In Anlehnung an Hinchcliffe und Kim, der Social Business als "[t]he Strategic Application of Social Computing to Enterprise Challenges" [2] beschreiben, definieren wir Social Business wie folgt:

Social Business wird in der vorliegenden Publikation allgemein als Strategie und Rahmenwerk verstanden, mit dessen Anwendung die Generierung eines sozialen, ökologischen und ökonomischen Nutzens aus dem Einsatz sozialer Netzwerke als primäres Ziel verbunden wird.

In der Betrachtung von Social Business als ganzheitliches Konzept wurden die Rahmenbedingungen und erweiterten Wertschöpfungsketten vor dem Hintergrund einer attraktiven Arbeitsgestaltung im Projekt untersucht. Im Ergebnis wurden Strategien und Konzepte zur proaktiven Arbeitsgestaltung im Kontext sozialer Netzwerke entwickelt, welche in einem Referenzmodell zusammengetragen und durch eine umfangreiche Pilotierung ergänzt wurden. Hierbei wurden folgende Schwerpunkte verfolgt:

- Weiterbildung und Wissensaustausch: Gestaltung digitalisierter Weiterbildung und Wissensaustausch mittels sozialer Netzwerke

- Partizipation und Innovation: Nutzung und Evaluation sozialer Netzwerke als Auslöser für Partizipation und Innovation

- Ökologie und Arbeitszeit: Entwicklung und Anwendung nachhaltiger Mobilitätskonzepte - wie soziale Netzwerke helfen ökologische und arbeitszeittechnische Aspekte zu verbinden

- Orientierung und Motivation: Anwendung und Evaluation von Social Business Orientierung und Motivation durch soziale Netzwerke

Ziel dieses Beitrags ist eine abschließend auf der Analyse der Anwendungsbeispiele beruhende kritische Auseinandersetzung mit dem Social Business Konzept. Dabei soll der Frage nachgegangen werden, mit welchen Herausforderungen beim Transformationsprozess sich Unternehmen in der Praxis konfrontiert sehen und wie diesen zu begegnen ist.

\subsection{Social Business Framework}

Innerhalb des SB:Digital Projektes wurde ein Rahmenwerk in Form eines Referenzmodells entwickelt, dieses diente zunächst der Orientierung und Schwerpunktdefinition im Forschungsprojekt. Im weiteren Projektverlauf wurde das Referenzmodell ausgebaut und stellt nunmehr eine praktische Orientierungshilfe für Unternehmen dar. Das Social Business Referenzmodell Abb. 24.1 beinhaltet eine Beschreibung des Social Business Transformationsprozesses, die Ausarbeitung eines Rollenmodells sowie eines Reifegradmodells und einen Leitfaden für den Einsatz von Social Business. 


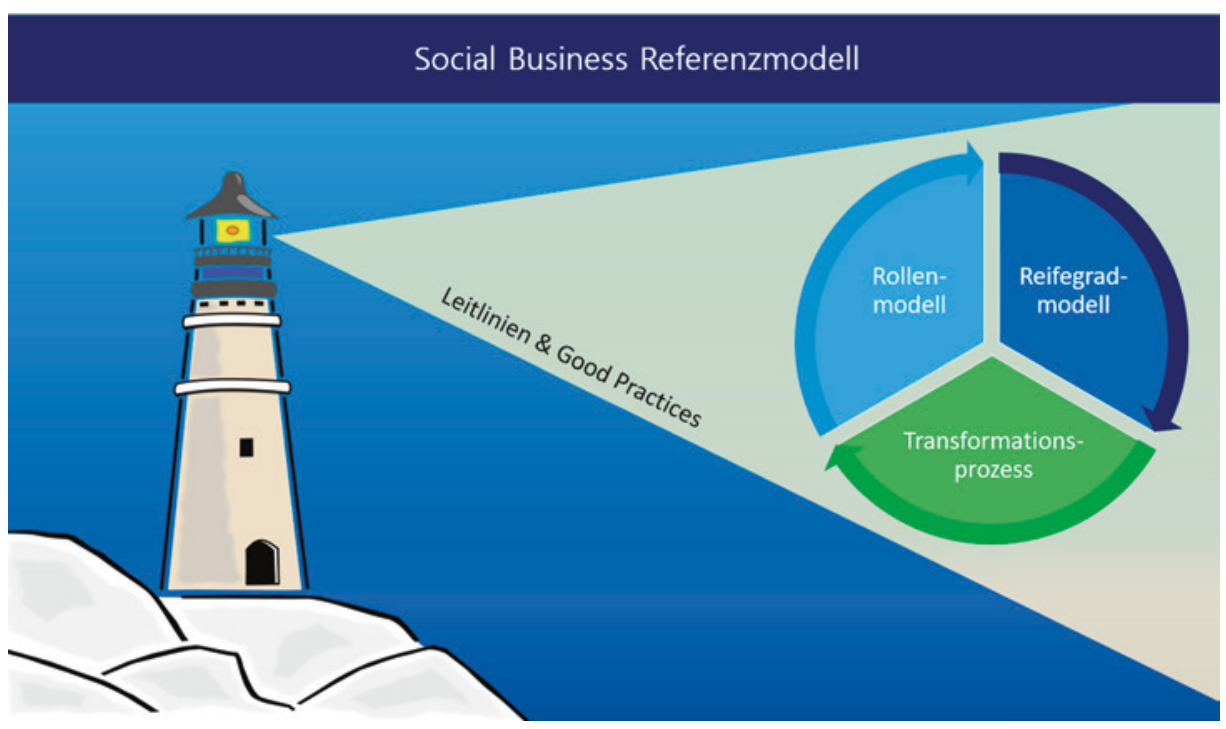

Abb. 24.1 Social Business Referenzmodell

\subsubsection{Transformationsprozess}

Den ersten und grundlegendsten Teil des Referenzmodells bildet ein vier Schritte umfassender Transformationsprozess Abb. 24.2. Dabei handelt es sich jedoch ausdrücklich nicht um einen linearen, sondern vielmehr einen iterativen Prozess, der sich je nach Anpassungsnotwendigkeit teilweise oder in einzelnen Schritten wiederholt.

Ausgangspunkt für die Social Business Transformation ist eine umfassende Analyse des Status quo auf Basis des Social Business Reifegradmodells. Grundlage für diese Analyse bilden die Arbeiten rund um die Mensch-Technik-Organisationsanalyse [10], welcher zur Untersuchung sozio-technischer Systeme verwendet werden können. Ziel der Ermittlung des Reifegrades ist es, Schwachstellen aufzudecken, die einer erfolgreichen Transformation und einer Übernahme der Social Business-Strategie im Wege

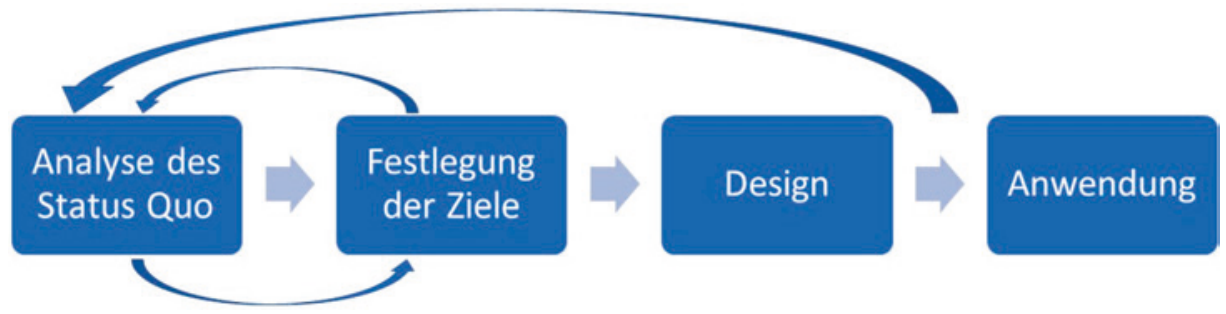

Abb. 24.2 Social Business Transformationsprozess 
stehen. So kann ein Unternehmen beispielsweise viel Aufwand in die Entwicklung einer technologischen Architektur gesteckt haben, ohne ein Bewusstsein für Social Business zu schaffen, was die Erfolgschancen der Initiative erheblich mindert.

In einem zweiten Schritt sind Ziele zu definieren (unter Berücksichtigung der aktuellen Situation). Dies ist unerlässlich, um die richtigen Prozesse und Aktivitäten, wie z. B. Sensibilisierungskampagnen einzuleiten, aber auch um die technologischen Instrumente und Indikatoren zur Erfolgsmessung auszuwählen. Eine Begrenzung der Ziele ist dabei nicht zwingend notwendig. Unternehmen können ein besseres Wissensmanagement anstreben und gleichzeitig eine Nachhaltigkeitskultur innerhalb des Unternehmensverbundes etablieren wollen. Was auch immer die Ziele eines Unternehmens sein mögen, es muss verstanden werden, dass die Transformation in Richtung Social Business kein linearer, sondern ein iterativer Prozess ist und Ziele nacheinander erreicht werden können bzw. sich zum Teil durch ihre Realisierungen gegenseitig befruchten. Dies bedeutet, dass die Ziele im ersten Anlauf möglicherweise nicht vollständig erreicht werden oder einzelne Ziele aufgrund sich ändernder Rahmenbedingungen angepasst werden müssen.

Während die ersten beiden Prozessschritte als theoretische Grundlage oder Anfangsphase verstanden werden können, beginnt im dritten Transformationsschritt die Designphase. Hierbei sollten wiederum die drei Ebenen Individuum, Organisation und Technologie berücksichtigt werden. Aus technologischer Sicht ist es entscheidend, dass soziale Netzwerktechnologie entsprechend den formulierten Zielen und Anforderungen zu entwerfen sind. Bestimmte Punkte sollten im Designprozess auf technologischer Ebene Berücksichtigung finden, z. B.:

- Erarbeitung eines Rechteverwaltungskonzeptes mit entsprechenden Rollen

- Definition und regelmäßige Überprüfung von Datenschutzrichtlinien, Einhaltungsund Löschungsregeln

- Planung von Feedback-Kanälen

- Prüfung von Integrationsmöglichkeiten und Bewertung ihrer Eignung

- Festlegung einer Umsetzungsstrategie

Auf organisatorischer Ebene können bestimmte Rahmenbedingungen, wie z. B. die Arbeitsgestaltung [4] oder eine Empowermentkultur [11], die eine erfolgreiche Umsetzung von Social Business unterstützen. Grundsätzlich muss der/die Mitarbeiter*in als zentrales Element im Social Business verstanden werden, das den Prozess der Zusammenarbeit am Laufen hält und mit dessen Handeln trägt. Ein offener Führungsstil, der die Mitarbeiter nicht durch hierarchische Strukturen einschränkt, sondern ihnen Selbstbestimmungsrechte gewährt, gilt als förderlich für Social Business [9]. Weitere relevante Aspekte auf der Organisationsebene sind:

- Entwicklung von Beteiligungsmechanismen und Transparenz

- Identifikation von Akteuren und Verantwortlichkeiten 
- Formulierung von Kommunikationsstrategie und -richtlinien

- Identifizierung von Wissensflüssen

- Planung der Erfolgskontrolle, Entwicklung von Kennzahlen und Parametern

Auf der individuellen Ebene muss klar sein, dass proportional zur Vernetzung und sozialen Interaktion die Flexibilität der Arbeit und die Notwendigkeit von Selbstbestimmung steigen. Es ist daher notwendig, die Beschäftigten in die Lage zu versetzen, selbstbestimmt und frei im sozialen Netzwerk zu agieren, damit sie sich selbst als die relevanten Akteure zu verstehen, die sie sind. Dies bringt neue Anforderungen, gleichzeitig, aber auch neue Chancen für Motivation und Wachstum. Um die Mitarbeiter für diese neue Arbeitsweise zu sensibilisieren, können Schulungs- oder Kommunikationskampagnen im Unternehmen notwendig sein. Neben diesen Maßnahmen bedeutet Social Business Design:

- Identifizierung von Kompetenzen und Einrichtung von Ausbildungsmechanismen

- Positive Rahmung von Mitarbeitern und Arbeitsgruppen

- Aufzeigen von Vorteilen und Nutzen

Dies zeigt, dass sich der Gestaltungs- und Transformationsprozess nicht auf die technologische Perspektive beschränken darf, sondern Rahmenbedingungen sowie individuelle Bedürfnisse und Anforderungen berücksichtigt werden müssen.

Schließlich wird der theoretisch geplante und entwickelte Ansatz zur Anwendung gebracht. Dabei ist ein durchgehendes Evaluationsmanagement erforderlich, sowohl vonseiten der Geschäftsführung als auch von anderen Beteiligten, wie zum Beispiel den Geschäftspartner*innen oder den Mitarbeitenden. Dabei spielt auch das Sammeln von Feedback eine zentrale Rolle, wodurch der Vorgang kontrolliert und, wenn nötig, stets angepasst werden kann.

\subsubsection{Reifegradmodell}

Um Unternehmen in die Lage zu versetzen, den aktuellen Stand der Bereitschaft zum Social Business zu ermitteln, wurde im Projekt ein Reifegradmodell entwickelt. In der Analyse werden die verschiedenen Bereiche des Unternehmens oder der Organisation einzeln nach ihrer „Social Business Reife“ bewertet, wodurch ein umfassendes Bild des Status quo gegeben werden kann. Hierbei werden die Aspekte Technik, Organisation und Mensch abgesteckt und entsprechend relevante Dimensionen genauer betrachtet, um darauf aufbauend die nächsten Schritte planen zu können.

Das Reifegradmodell umfasst die in Abb. 24.3 aufgeführten fünf Dimensionen und gibt in vier Stufen deren Reifezustand an. Ergänzt wird das Modell durch sogenannte Einflussfaktoren, die ebenfalls entscheidend für die Bewertung der Social Business Reife eines Unternehmens sind, obgleich sie nicht in dezidierten Abstufungen bewertet werden können. 


\begin{tabular}{|l|l|l|l|l|}
\hline DIMENSION & REIFEGRAD & Problem- \\
\hline BEWUSSTSEIN & Fehlend & $\begin{array}{l}\text { Prozess- } \\
\text { bewusstsein }\end{array}$ & Verantwortung \\
\hline FÄHIGKEITEN & $\begin{array}{l}\text { Keine SB- } \\
\text { Fähigkeiten }\end{array}$ & Kerstehen & Partizipieren & Netzwerken \\
\hline ROLLEN & Keine SB-Rollen & $\begin{array}{l}\text { Marketing- } \\
\text { getrieben }\end{array}$ & Informell & $\begin{array}{l}\text { Explizit definiert } \\
\text { und zugewiesen }\end{array}$ \\
\hline KOLLABORATION & Ad hoc & Team-weit & $\begin{array}{l}\text { Unternehmens- } \\
\text { weit }\end{array}$ & Im Netzwerk \\
\hline $\begin{array}{l}\text { SOCIAL BUSINESS } \\
\text { INFRASTRUKTUR }\end{array}$ & $\begin{array}{l}\text { Keine sozialen } \\
\text { Technologien }\end{array}$ & $\begin{array}{l}\text { Externe } \\
\text { Anwendungen }\end{array}$ & ESN & $\begin{array}{l}\text { Social Software } \\
\text { vollständig } \\
\text { integriert }\end{array}$ \\
\hline
\end{tabular}

Abb. 24.3 Das Social Business Reifegradmodell

Bestimmte Rahmenbedingungen, wie z. B. die Arbeitsgestaltung, sind für die erfolgreiche Umsetzung von Social Business von Vorteil. Auch muss klar sein, dass für Vernetzung und soziale Interaktionen Arbeitszeit kalkuliert werden muss und dass flexiblere Arbeitsbedingungen und ein höheres Maß an Selbstbestimmung neue Anforderungen an die Unternehmenskultur mit sich bringen. Durch die Ermittlung des aktuellen Reifezustandes des Unternehmens können Stärken und Schwachstellen ausgemacht werden und darauf aufbauend Ziele und Strategien festgelegt werden.

\subsubsection{Social Business Rollenmodell}

Im Verlauf der Projektarbeit wurde deutlich, dass der erfolgreiche Einsatz von Social Business einer klaren Zuschreibung von Verantwortlichkeiten bedarf. Zu diesem Zweck wurde ein Social Business Rollenmodell entwickelt, welches aus empirischen Studien, Experteninterviews und Umfragen entwickelt wurde [7]. In Zusammenarbeit mit ICU (Internes Crowdsourcing in Unternehmen), einem Partner aus dem TransWork Verbundprojekt, wurde diese Modell validiert [14]. Schließlich umfasst das Rollenmodell sechs Rollen: (1) Social Business Manager, (2) Content Manager, (3) Developer, (4) Communication Manager, (5) Community Stakeholder, (6) Executive.

Diese wurden als die zentral wichtigen Rollen für die erfolgreiche Entwicklung und Umsetzung einer Social Business Strategie identifiziert. Jeder Rolle sind verschiedene Zuständigkeits- und Aufgabenbereiche sowie dazugehörige Qualifikationen und Kompetenzen zugewiesen. Dabei ist hervorzuheben, dass die definierten Rollen nicht an einzelne Personen gebunden sein müssen und z. B. eine Rolle von mehreren Personen ausgeführt werden kann. Auch kann eine Person in mehreren Rollen parallel eingesetzt sein. Über die Definition der Relationen zwischen den Rollen konnten außerdem die Zuständigkeitsbereiche besser abgegrenzt und Kollaborationsbereiche aufgezeigt werden. 


\subsubsection{Leitfaden und Good Practices}

Die im Projektverlauf gewonnen Erkenntnisse wurden in eine umfassende Sammlung von Empfehlungen integriert, die in der Implementierung von Social Business von großer Hilfe sein können. Dabei wurden zunächst in einem Leitfaden verschiedene Punkte gesammelt, genauso wie "Good-Practices", die aus verschiedenen Praxisanwendungen stammten. Diese Punkte wurden während der Projektlaufzeit weiterentwickelt und sind nun in Form von konkreten Handlungsempfehlungen zusammengefasst. Diese sind auch im Schlussteil dieses Beitrages zu finden.

\subsection{Social Business in Action}

Die Realisierung ihres jeweiligen Social Business Konzeptes in der Praxis stellte die am SB:Digital Projekt beteiligten Unternehmen vor unterschiedlichste Herausforderungen. Im Folgenden sollen die Projekte und deren jeweilige Herausforderungen jeweils in Kürze skizziert sowie klassifiziert werden und auf Lösungsansätze eingegangen werden.

\subsubsection{Pilotanwendung Orientierung und Motivation}

Das Ziel der Pilotanwendung Orientierung und Motivation war es, gezielt Gestaltungsparameter für ein Social Business zu ermitteln und zu untersuchen, wie Mitarbeitende am effektivsten zur Nutzung eines ESN motiviert werden können. Die Vertriebs- und Entwicklung mbh CADsys bringt als Systemhaus für Softwarelösungen und -entwicklungen, die idealen Voraussetzungen für Social Business mit. Kommunikation, sowohl intern als auch mit dem Kunden stellt einen wichtigen Baustein im Unternehmen dar. Die Analyse des Status quo zeigte jedoch, dass die Arbeitsprozesse durch brüchige Informations- und Kommunikationsketten sowie einen hohen organisatorischen Aufwand gestört waren. Das Unternehmen war somit bestrebt, die Kommunikationsabläufe zu vereinfachen. Zugleich sollten Wissensmanagementaktivitäten wie das Zusammentragen von Informationen und das Ausformulieren bzw. das Niederschreiben von bislang nur implizit vorhandenem Wissen motivierender gestaltet werden. Auf diese Weise sollte eine bessere Einbindung der Belegschaft erreicht werden. Des Weiteren wurde die Generierung eines künftig offenen Informationsaustausches verfolgt, um das Suchen und Finden von Informationen/Wissen effizienter zu gestalten. Folgende Zielstellungen sollten konkret mit der Implementierung erreicht werden:

- verbesserte Einarbeitung neuer Mitarbeitenden

- schnellere und effizientere Kommunikationswege

- einfache Bereitstellung relevanter Dokumente

- Zeitersparnis, durch den Wegfall zeitraubender Informationssuchen 
- verbesserte Zusammenarbeit

- Aufbau eines kollegialen Wir-Gefühls

Das Design des Lösungsansatzes sah deshalb vor, ein kollaboratives Wissensmanagement aufzubauen, welches die Orientierung im Unternehmen insbesondere im Hinblick auf das Onboarding erleichtert und die Motivation am zielgerichteten Arbeiten aufrechterhalten sollte. Als technische Grundlage entschied sich das Unternehmen für einen internen Blog. Ein Corporate Blog kann sowohl für die interne als auch externe Kommunikationen und den Wissensaustausch genutzt werden und entsprach somit den Zielsetzungen des Unternehmens, wonach es neben einem internen kollaborativen Wissensmanagement auch einen externen Bereich (zur Kundenkommunikation) geben sollte.

In der Realisierung des kollaborativen Wissensmanagements zeigten sich folgende zentrale Herausforderungen:

- Systemauswahl und Bedienbarkeit des Tools: Bereits die Auswahl und Gestaltung eines an den Bedarfen ausgerichteten ESN stellte das Unternehmen vor Herausforderungen. Das anfänglich als Blog konzipierte System wurde später durch ein kostenfreies Content Management System ersetzt, da es als Open Source die nötige technische Grundlage bot und abgesehen vom Arbeitsaufwand keine weiteren Kosten generierte. Die Herausforderung in der Gestaltung des Autorentools bestand insbesondere in der Bereitstellung eines einfach $\mathrm{zu}$ erlernenden und bedienbaren Userinterfaces, Möglichkeiten zur Erstellung optisch ansprechender Inhalte sowie der Lauffähigkeit als Web-Applikation mit minimalen Administrationsaufwand. Im Projekt begegnete man diesen Herausforderungen durch eine Testreihe verschiedener Systemdesigns. Faktoren, die hier eine Rolle spielten, waren das Branding, Layout und Stylesheets.

- Anhaltende Motivation der Mitarbeiter zur Nutzung des Systems: Nach der Implementierung des digitalen sozialen Netzwerkes zeigte sich nach geraumer Zeit eine recht verhaltende Nutzung durch die Belegschaft. Während der Einstiegsphase setzte die Geschäftsleitung auf einen Schulungsworkshop und die anschließende Entwicklung einer Eigendynamik. Auf die Implementierung von Anreizsystemen (bspw. durch Gamification) wurde zunächst verzichtet. Um den bestehenden Barrieren zu begegnen, werden inzwischen jedoch mögliche Anreize, wie die Erhöhung des Personalisierungsgrades, Designanpassungen und verbesserte Feedback-Darstellungen diskutiert.

\subsubsection{Pilotanwendung Ökologie und Arbeitszeit}

Im Gegensatz zur ersten, vorranging innerbetrieblich genutzten Pilotanwendung stand bei der Entwicklung einer Anwendung für Ökologie und Arbeitszeit die Etablierung des 
Social Business Ansatzes in sehr großen Unternehmen mit einem zentralen Standort oder lokalen Unternehmensverbünden (bspw. Gewerbepark) im Fokus. Das Unternehmen highQ Computerlösungen strebte dabei insbesondere die Entwicklung und Anwendung nachhaltiger Mobilitätskonzepte an. Entsprechend der Vision sollte ökologische und arbeitszeittechnische Aspekte auf Basis digitaler sozialer Netzwerke miteinander verbunden werden.

Der zuvor skizzierte Transformationsprozess wurde im Teilprojekt Ökologie und Arbeitszeit modifiziert. Ausgehend von der gesellschaftlichen Herausforderung (Klimawandel, Starke Feinstaubbelastung der Ballungsräume, etc.) wurde das Ziel formuliert mithilfe eines digitalen sozialen Netzwerkes, Anreize für umweltfreundliches Mobilitätsverhalten zu schaffen. Mit dieser Zielstellung wurde eine unternehmensunabhängig einsetzbare Mobilitätsapp (mytraQ.biz) konzipiert und umgesetzt. Mit dieser Anwendung wurde im Rahmen von SB:Digital eine neuartige Form eines Unternehmensnetzwerkes entwickelt, die ihren Nutzerinnen und Nutzern durch software-gestützte Empfehlungen von Stau vermeidenden Pendlerwegen und -zeiten die Etablierung flexibler Arbeitszeitmodelle im Unternehmen bzw. im Unternehmensverbund ermöglicht. Konkrete Vorteile von mytraQ.biz sind:

- Berechnung möglicher Pendelwege (z. B. Entzerrung der Arbeitszeiten, um Stauzeiten zu vermeiden, Nutzung nachhaltiger Mobilitätsangebote)

- Kommunikation der Mitarbeitenden einer Organisation (geschlossene Nutzergruppe) zur Optimierung täglicher Pendelwege (z. B. carpooling, trafficjams, etc.)

- Integration von Incentives für ressourcenschonendes Verhalten (z. B. gestaffelte Zeitmeilen fürs Radfahren, ÖPNV-Nutzung, Carsharing)

- Motivation für ökologisch nachhaltiges Verhalten durch Gamification-Ansätze (z. B. virtuelle Storyline mit integriertem Rewardsystem)

- Organisationsumfassendes Reporting zum Verkehrsverhalten (anonymisiert)

Die Stärke der Anwendung ist die Etablierung eines überbetrieblichen Mobilitätskonzeptes, welches eine Antwort auf die mit täglichen Pendlerströmen verbundenen infrastrukturellen und ökologischen Probleme bietet. In der Pilotkundenakquise erweist sich dies jedoch als Hindernis, da die Varianz unterschiedlicher Interessen (ökologischer Footprint des Unternehmens, infrastrukturelle Ausgaben, Senkung des Stresspegels von Mitarbeitenden) von Unternehmen und Nutzergruppen eine gezielte Ansprache und damit eine erfolgreiche Kundenakquise erschwert. Obgleich das Bewusstsein für die gesellschaftlichen Herausforderungen (Klimawandel etc.) vorhanden ist und das Konzept einer auf ökologischer und sozialer Ebene entlastenden Pendlermobilität potenzielle Pilotkunden begeistert, gab es seitens der Pilotkunden verschiedene Vorbehalte:

- Vertrauen: Die Erhebung und Auswertung personenbezogener Mobilitätsdaten der vorgeschlagenen Anwendung ist ein sehr kritisches Element und erweist sich als zentrale Barriere. Obwohl die Anwendung transparente DSGVO-Konformität besitzt, 
herrscht eine gewisse Skepsis - Arbeitgeber*innen befürchten zur Verantwortung gezogen zu werden und Arbeitnehmer*innen befürchten Überwachungsmöglichkeiten und mögliche Mechanismen der Kontrolle - sowie den Verlust der Datensouveränität. Hauptursache dieser Skepsis ist fehlendes Vertrauen zwischen vielen Parteien - nicht nur zwischen Anbieter und Kunden (Unternehmen). Dabei wird deutlich, dass das im Hinblick auf Social Business notwendige Vertrauen zwischen den vertretenen Interessensgruppen in Unternehmensverbünden (Arbeitgeber, Arbeitnehmervertretung, Marketingabteilungen etc.) häufig nicht gegeben ist. Wie in allen Ausprägungen von Social Business ist dies jedoch ein zentraler Erfolgsfaktor.

- Strukturen und Prozesse: Die durch soziale Netzwerke vorangetriebene Lockerung formaler Strukturen stößt insbesondere in Unternehmen mit strengen Hierarchien auf Widerstand. Ein soziales Netzwerk in dem der Chef Fahrgemeinschaften mit seinen Mitarbeitenden bildet ist in einigen Köpfen schier nicht denkbar.

Bei dem im Projekt entwickelten sozialen Netzwerk handelt es sich um eine völlig neue Form von Kooperationsmodell und bringt entsprechende Herausforderung mit sich. Anders als bei vielen ESN ist der Zweck der Vernetzung nicht primär ökonomisch, sondern ökologisch getrieben - was jedoch auch ökonomischen Nutzen einschließt (direkt und indirekt). Der Kooperation der beteiligten Unternehmen und der Interessensgruppen muss ein Konsens über diese Zielsetzung zugrunde liegen. Der Nutzen, die Zielstellung sowie die konkreten Nutzungsszenarien müssen verstanden und als wichtig eingestuft werden - ein Bewusstsein muss geschaffen werden.

\subsubsection{Pilotanwendung Partizipation und Innovation}

Mit dem Teilprojekt Partizipation und Innovation wurde das Ziel einer Untersuchung der Auswirkungen und Potenziale von sozialen Netzwerken auf die innerbetriebliche Zusammenarbeit und Partizipation verfolgt. In Kooperation mit dem Unternehmen unymira USU GmbH, dem führenden Anbieter für IT- und Knowledge-ManagementSoftware, wurde das Social Media Servicemanagement-Tool Connect entwickelt und dessen Einsatz im Anwendungsfall erprobt. In der Grundkonfiguration dient dieses Werkzeug dazu, verschiedene Social Media und Messaging Kanäle in einem zentralen Tool organisieren zu können. Auf diese Weise wird ermöglicht, eine Multi Channel Kommunikationen an einem Arbeitsplatz zu zentrieren und übersichtlich zu organisieren. Damit zielt das Tool insbesondere auf den Einsatz in Unternehmen mit einem breit angelegten Kundendienst ab. Die Bündelung verschiedener Medienkanäle bietet die Gelegenheit, die Kommunikation zwischen den Kunden und den Mitarbeitenden moderativ zu steuern. Die Erforschung der Potenziale zur Weiterentwicklung des Tools im Rahmen des Projektes zielte darauf ab, das Innovationspotenzial der gesamten Belegschaft zu nutzen, zu fördern und deren allgemeine Motivation sich einzubringen, zu steigern. Themenspezifische Probleme und Kundenanfragen würden durch einen Bot 
nach Schlagwörtern gefiltert und könnten somit von der jeweiligen fachkundigen Person direkt und zeitnah beantwortet werden. In der Umsetzung zeigten sich verschiedene Herausforderungen, die leider nur teilweise beseitigt werden konnten:

- Technische Herausforderungen: Durch die während dem Projekt veränderten Ansprüche an das System, wurden auch systemtechnische Anforderungen bezüglich der Gestaltung einer bedarfsgerechten Software, d. h. die sichere Zusammenführung interner und externer Netzwerke komplexer. Diesen Anforderungen konnte jedoch im Laufe des Projektes erfolgreich durch die Integration verschiedener technischer Sicherungsinstanzen begegnet werden.

- Datenschutz und Vertrauen: Eine zentrale Herausforderung lag in der Schaffung einer DSGVO-Konformität sowie der Unternehmens-Compliance. Durch die 2018 wirksam gewordene Verschärfung der Datenschutz-Grundverordnung bremsen sowohl Management als auch Interessensvertreter*innen einen fortschreitenden Transformationsprozess häufig aus. Ursache sind Skepsis und Unsicherheit hinsichtlich systemischer Funktionsweisen sowie die Befürchtung öffentlicher Skandale, falls sensible Daten öffentlich werden. Das Risiko wurde an dieser Stelle als hoch wahrgenommen - obwohl die technischen Risiken als eher niedrig einzustufen waren. Aufgrund dessen wird auf unternehmenssteigernde Potenziale, wie Automatisierung zeitaufwendiger Koordinationsprozesse, Vertrauensbildung durch direkte qualifizierte Kundenkommunikation oder die Ausschöpfung unternehmensweiter Ressourcen für neue Innovationsprozesse, verzichtet.

Allgemein lässt sich feststellen, dass die Schaffung einer Schnittstelle zwischen Internet und Intranet für Unternehmen und Führungseben oft als hohes Risiko für die Unternehmensprozesse eingestuft und somit die in der Anfangsphase des Transformationsprozesses erkannten Potenziale gegenüber den Risiken, wie etwa externe Angriffe auf Unternehmensdaten, als nicht tragbar erachtet werden. Zwar wurden im Rahmen des Projektes Maßnahmen zur Lösung der Datenschutzproblematik erarbeitet, diese erweisen sich jedoch bis dato als ein zu hoher finanzieller und zeitlicher Einsatz. Daher obliegt es jedem Unternehmen selbst das Risiko gegenüber seiner Wettbewerbsfähigkeit abzuwägen.

\subsubsection{Pilotanwendung Weiterbildung und Wissensaustausch}

Die Gestaltung einer digitalisierten Weiterbildungsanwendung und Etablierung eines verbesserten Wissensaustausches mittels sozialer Netzwerke stand im Fokus der vierten Pilotanwendung. Durch die soziale Interaktion im spielerischen Wettbewerb und punktgenaue Lerninhalte, die basierend auf Echtzeitanalysen aus der Spielsituation heraus bereitgestellt werden, soll die Motivation zur Weiterbildung hochgehalten und der Lernerfolg nachhaltig gestaltet werden. Die Pilotanwendung yeepa ${ }^{\circledR}$ stützt sich auf drei Säulen, die den Lernzyklus definieren: Spielen, Messen, Lernen. 
Dabei setzt yeepa ${ }^{\circledR}$ auf das Prinzip der Berechnung von Wissenskapital, dargestellt in Form des yeepa-Index, und ist in der Lage, kontinuierlich eintreffende Spieldaten auf der mathematischen Grundlage der Item Response Theory (IRT) zu analysieren und Spielleistungen zu einem instruktiven Indexwert zu kondensieren.

Begreift man Lernen als sozialen Prozess [6], dann setzt yeepa ${ }^{\circledR}$ mit der psychometrischen Validierung von Kommunikations- und Lernzielen an folgenden Punkten an:

- Intrinsische Motivation: spielerischer Wettbewerb mit anderen

- Extrinsische Motivation: soziale Peer-Effekte (Interaktion), Preise

- Selbsterfahrung: Wissensstandmessung

- Türöffner-Setting: Anregung sachorientierter (Offline-)Diskussionen unter Nutzern

- Selbststeuerung: selbstbestimmtes Spielen

Im Verlauf des Projektes konnte yeepa ${ }^{\circledR}$ erfolgreich in zahlreichen überbetrieblichen Settings, insbesondere im Bereich der Erwachsenenbildung in internationalen Wissenswettbewerben, eingesetzt werden. Dadurch konnte die Entwicklung eines Analytic Dashboards als Feedbackmechanismus maßgeblich vorangetrieben werden [12]. Im Einsatz von yeepa ${ }^{\circledR}$ in internationalen Wissenswettbewerben zeigte sich zudem, dass sowohl die technischen Hürden (als mobile und Web-App) als auch die Motivation der Nutzer dank der eingebauten Spieldynamik keine wesentliche Hürde bei der Implementierung darstellt. Die größte Herausforderung für den innerbetrieblichen Einsatz des Social Learnings in ESN stellt vielmehr die Spielumgebung dar. Als die bedeutendsten Herausforderungen kristallisierten sich folgende Punkte heraus:

- Unternehmenskultur: In den zahlreichen Untersuchungsgesprächen, Workshops und Veranstaltungen mit verschiedenen deutschen Großunternehmen zeigte sich, dass die Standardprozesse bezüglich der Auswertung von Aus- und Weiterbildung noch nicht auf dem Stand moderner analytischer Verfahren sind. Vor allem HR-Abteilungen sind zum Teil weit entfernt von einer prozess-integrierten Erfassung von Kompetenzen und Wissen. Gleichzeitig ist wenig Bewusstsein für den Nutzen und die Chancen individuellen Lernens und Weiterbildungsangeboten jenseits des Standardkatalogs vorhanden.

- Skepsis seitens der Führungsebene: Eine auf Analytik beruhende Wirkungsmessung für den innerbetrieblichen Einsatz stößt derzeit häufig noch auf allgemeine Skepsis (u. a. Datenschutzbedenken). Während in überbetrieblichen Netzwerken sowie bei Unternehmen mit internationalen Weiterbildungsprogrammen der Aufwand zur Identifikation geeigneter Wissensbereiche und zur Integration von Inhalten in Form von Quiz-Fragen und Lerneinheiten angemessen erscheint, scheuen Unternehmen, die einem geringeren Anpassungsdruck unterliegen den Aufwand für solch innovative Angebote. Gleichzeitig herrschen zum Teil große Vorbehalte gegenüber Serious Games - die Mitarbeitenden seien ja schließlich nicht zum Spielen da. 


\subsection{Allgemeine Handlungsempfehlungen}

Die im Rahmen des Projektes erforschten Anwendungsszenarien verdeutlichen die vielseitigen Potenziale von Social Business. Gleichzeitig wird der Blick für den weiteren Handlungsbedarf zur konzeptionellen Erforschung von Leitlinien zur Unterstützung von Unternehmen auf ihrem Weg zum Social Business geschärft. Die in der Praxis erlebten Herausforderungen machen deutlich, dass es sich bei der Implementierung von Social Business im Grunde ein Aushandlungsprozess handelt. In diesen Prozess müssen alle unternehmensbezogenen Ebenen einbezogen werden. Aufgrund der hohen Variantenvielfalt bei Unternehmen, Anwendungsbereichen von ESN sowie unterschiedlich fortgeschrittenen Reifegraden wird an dieser Stelle versucht, möglichst allgemeingültige Lösungsansätze sowie Handlungsempfehlungen zur Begegnung der Herausforderungen zu geben (Abb. 24.4).

\subsection{Fazit}

Ziel des Projektes SB:Digital war es, die Grenzen der Forschung und Entwicklung rund um ESN, digitale Kollaboration, Enterprise 2.0 und Social Business neu auszuloten. Social Business verstanden als Konzept welches ökonomischen, ökologischen und sozialen Nutzen schaffen kann, geht über das bis dato bekannte Wissen über die Wirksamkeit von

\begin{tabular}{|c|c|c|}
\hline & Herausforderungen & Handlungsempfehlungen \\
\hline \multirow{4}{*}{ 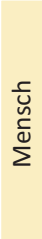 } & \multirow{2}{*}{$\begin{array}{c}\text { Skepsis gegenüber } \\
\text { Empowerment-Prozessen }\end{array}$} & Nutzen und Potentiale aufzeigen \\
\hline & & Wirkungsweise und Mechanismen demonstrieren \\
\hline & Skepsis gegenüber Technik & Mitarbeitende in den Transformationsprozess einbinden \\
\hline & Geringe Motivation & Scheitern als Teil des Transformationsprozesses akzeptieren \\
\hline \multirow{4}{*}{ 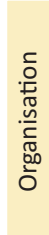 } & $\begin{array}{l}\text { Starre, technikfeindliche } \\
\text { Unternehmenskultur }\end{array}$ & Anreizsysteme gestalten \\
\hline & Fehlende Strukturen \& Prozesse & $\begin{array}{l}\text { Rollenzuweisung zur Zuordnung von Aufgaben und } \\
\text { Zuständigkeiten }\end{array}$ \\
\hline & \multirow[b]{2}{*}{ Skepsis gegenüber ROI } & Klare Zielstellungen definieren \\
\hline & & Prozess neu- oder umgestalten \\
\hline \multirow{2}{*}{ 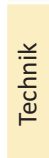 } & Schlechte Bedienbarkeit & $\begin{array}{l}\text { System- und Designanpassungen ( z.B. ansprechendes User } \\
\text { Interface, Benutzerhinweise, Feedback-Darstellungen, etc.) }\end{array}$ \\
\hline & $\begin{array}{l}\text { Risiken für Datenschutz \& } \\
\text { Sicherheit }\end{array}$ & Überprüfung der DSGVO-Konformität \\
\hline
\end{tabular}

Abb. 24.4 Handlungsempfehlungen auf Basis der Herausforderungen 
sozialen Netzwerken in Unternehmen und in Unternehmensverbünden weit hinaus. Das Projekt hat damit die Forschungslandschaft nicht nur um eine neue Sichtweise erweitert, sondern drüber hinaus erreicht, dass eine bessere Operationalisierung (siehe Reifegradmodell und Rollenmodell) des Phänomens Social Business vorliegt.

Die hier beschriebenen Herausforderungen und die vorgeschlagenen Lösungswege sind Teil dieser Forschung und helfen Unternehmen einen guten Weg zur digitalen Kollaboration zu finden - nicht nur in einem rein ökonomischen Interesse. Hierbei gibt es wenige signifikante Knackpunkte, welche immer wieder auffällig waren. (1) Das Thema Datenschutz, Datensicherheit und Datensouveränität ist so ein wichtiger Punkt. Hierzu zählen nicht nur die technischen Realisierungen (welche z. T. sehr hochwertig sind), sondern vor allem das Gefühl und die Kultur (also die gelebte Praxis des Datenschutzes) innerhalb des Unternehmens oder der Unternehmensverbünde. Ist eine Unternehmensführung (oder ein Anbieter) nicht in der Lage hier Klarheit und Transparenz zu schaffen, ist das Vorhaben Social Business quasi zum Scheitern verurteilt, weil nie der gesamte Nutzen aus einer freien Kommunikation gezogen werden kann. (2) Ein grundsätzliches Verständnis vom Nutzen des Einsatzes von sozialen Netzwerken sollte bei einer kritischen Masse innerhalb des Unternehmens vorhanden sein, welche die Entwicklungen treiben und den Nutzen klar kommunizieren können. (3) Eine zu geringe Beteiligung der Mitarbeitenden wird aus Sicht der Unternehmen häufig für das Scheitern von ESN genannt [8]. Die Aufrechterhaltung der Motivation der Belegschaft zu einem zielgerichteten Arbeiten, ist ohnehin eine alltägliche Herausforderung der Unternehmensführung. Um die Motivation zu einer nachhaltigen Nutzung sozialer Netzwerke im Unternehmenskontext zu steigern, ist schlussendlich auch hier eine klare transparente Kommunikation notwendig, um einerseits Ängste zu mindern und Vertrauen zu stärken. Ansprechende Systemdesigns sowie mögliche Anreizsysteme (z. B. Ratings oder Arbeitszeitgutschriften) können je nach Bedarf ebenfalls in Betracht gezogen werden.

\section{Projektpartner und Aufgaben}

- Institut für Angewandte Informatik e. V. (InfAI), Leipzig Entwicklung eines Rahmenkonzeptes zur Gestaltung der Arbeit von morgen mittels digitaler sozialer Netzwerke

- Fraunhofer-Institut für Arbeitswirtschaft und Organisation IAO, Stuttgart Analyse und Gestaltung sozialer Netzwerke - Entwicklung, Trends und Best Practices

- B.I.G Social Media GmbH, Berlin

Nutzung und Evaluation sozialer Netzwerke als Auslöser für Partizipation und Innovation

- CADsys Vertriebs- und Entwicklungsgesellschaft mbH, Chemnitz Anwendung und Evaluation von Social Business - Orientierung und Motivation durch soziale Netzwerke 


\section{- highQ Computerlösungen GmbH, Freiburg}

Entwicklung und Anwendung nachhaltiger Mobilitätskonzepte - wie soziale Netzwerke helfen, ökologische und arbeitszeittechnische Aspekte zu verbinden

- SNTL Publishing GmbH \& Co KG, Berlin

Gestaltung digitalisierter Weiterbildung und Wissensaustauschs mittels sozialer Netzwerke

\section{Literatur}

1. Hinchcliffe D, Kim P (2012) Social business by design. Transformative social media strategies for the connected company. Wiley, Hoboken

2. Kiron D, Palmer D, Phillips, Anh Nguyen, Kruschwitz N (2012) Social business. What are companies really doing? MIT Sloan management. https://deloitte.wsj.com/cfo/files/2012/07/ MITSloan_Deloitte-report.pdf. Zugegriffen: 17. Apr 2020

3. Koch M (2008) CSCW and Enterprise 2.0 - Towards an integrated perspective. BLED 2008 Proceedings 15. https://aisel.aisnet.org/bled2008/15

4. Krogh G v (2012) How does social software change knowledge management? Toward a strategic research agenda. J Strateg Inf Syst 21(2):154-164. 10.1016/j.jsis.2012.04.003

5. Michl W (2015) Erlebnispädagogik, 3., aktualisierte Auflage. UTB Profile, Band 3049. Ernst Reinhardt Verlag; UTB, München, Basel, München

6. Schiller C, Meiren T (2020) Rollen und Verantwortlichkeiten für erfolgreiche Social-BusinessAnwendungen. In: Daum M, Ulbrich H, Wedel M, Zinke-Wehlmann C (Hrsg) Gestaltung vernetzt-flexibler Arbeit. Beiträge aus Theorie und Praxis für die digitale Arbeitswelt. Springer Vieweg

7. Schiller C, Zinke-Wehlmann C (2019) Social Business. Studie über den Einsatz interner sozialer Netzwerke in Unternehmen, 1. Auflage. Fraunhofer Verlag, Stuttgart

8. Schönbohm R (2016) Enterprise Social Networks (ESN): Keimzelle agiler Unternehmen. In: Rossmann A, Stei G, Besch M (Hrsg) Enterprise Social Networks, Band 47. Springer Fachmedien Wiesbaden, Wiesbaden, S 247-275

9. Strohm O, Ulrich E (1997) Unternehmen arbeitspsychologisch bewerten. vdf, Hochschulverlag an der ETH Zürich, Zürich

10. Turban E, Strauss J, Lai L (2016) The Social Enterprise: From Recruiting to Problem Solving and Collaboration. In: Turban E, Strauss J, Lai L (Hrsg) Social Commerce. Springer International Publishing, Cham, S 181-203

11. Zinke C, Friedrich J, Haefner A (2018) Motivation for Corporate Training Through Feedback in Social Serious Games. In: 2018 IEEE International Conference on Engineering, Technology and Innovation (ICE/ITMC). IEEE, S 1-9

12. Zinke-Wehlmann C, Friedrich J (2019) Commute Green! The Potential of Enterprise Social Networks for Ecological Mobility Concepts. In: Camarinha-Matos L M, Afsarmanesh H, Antonelli D (Hrsg) Collaborative Networks and Digital Transformation, Band 568. Springer International Publishing, Cham, S 128-139

13. Zinke-Wehlmann C, Friedrich J, Römer V (2020) Power to the network. The concept of Social Business and its relevance for IC. In: Ulbrich H, Wedel M, Dienel H-xL (Hrsg) Internal Crowdsourcing in Companies. Theoretical Foundations and Practical Applications. Springer Nature, Berlin Dissertation 
Open Access Dieses Kapitel wird unter der Creative Commons Namensnennung 4.0 International Lizenz (http://creativecommons.org/licenses/by/4.0/deed.de) veröffentlicht, welche die Nutzung, Vervielfältigung, Bearbeitung, Verbreitung und Wiedergabe in jeglichem Medium und Format erlaubt, sofern Sie den/die ursprünglichen Autor(en) und die Quelle ordnungsgemäß nennen, einen Link zur Creative Commons Lizenz beifügen und angeben, ob Änderungen vorgenommen wurden.

Die in diesem Kapitel enthaltenen Bilder und sonstiges Drittmaterial unterliegen ebenfalls der genannten Creative Commons Lizenz, sofern sich aus der Abbildungslegende nichts anderes ergibt. Sofern das betreffende Material nicht unter der genannten Creative Commons Lizenz steht und die betreffende Handlung nicht nach gesetzlichen Vorschriften erlaubt ist, ist für die oben aufgeführten Weiterverwendungen des Materials die Einwilligung des jeweiligen Rechteinhabers einzuholen.

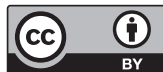

